\title{
Alien chromosome segment from Aegilops speltoides and Dasypyrum villosum increases drought tolerance in wheat via profuse and deep root system
}

\author{
M. Djanaguiraman ${ }^{1,7}$, P. V. V. Prasad ${ }^{1 *}$ (D, J. Kumari ${ }^{2}$, S. K. Sehgal ${ }^{3}$, B. Friebe $^{4}$, I. Djalovic ${ }^{5}$, Y. Chen $^{6}$, \\ K. H. M. Siddique ${ }^{6}$ and B. S. Gill ${ }^{4}$
}

\begin{abstract}
Background: Recurrent drought associated with climate change is a major constraint to wheat (Triticum aestivum L.) productivity. This study aimed to (i) quantify the effects of addition/substitution/translocation of chromosome segments from wild relatives of wheat on the root, physiological and yield traits of hexaploid wheat under drought, and (ii) understand the mechanism(s) associated with drought tolerance or susceptibility in wheat-alien chromosome lines.

Methods: A set of 48 wheat-alien chromosome lines (addition/substitution/translocation lines) with Chinese Spring background were used. Seedling root traits were studied on solid agar medium. To understand the influence of drought on the root system of adult plants, these 48 lines were grown in 150-cm columns for $65 \mathrm{~d}$ under full irrigation or withholding water for $58 \mathrm{~d}$. To quantify the effect of drought on physiological and yield traits, the 48 lines were grown in pots under full irrigation until anthesis; after that, half of the plants were drought stressed by withholding water for $16 \mathrm{~d}$ before recording physiological and yield-associated traits.

Results: The alien chromosome lines exhibited altered root architecture and decreased photochemical efficiency and seed yield and its components under drought. The wheat-alien chromosome lines T5DS.5S\#3L (TA5088) with a chromosome segment from Aegilops speltoides (5S) and T5DL:5 V\#3S (TA5638) with a chromosome segment from Dasypyrum villosum ( $5 \mathrm{~V}$ ) were identified as drought tolerant, and the drought tolerance mechanism was associated with a deep, thin and profuse root system.

Conclusions: The two germplasm lines (TA5088 and TA5638) could be used in wheat breeding programs to improve drought tolerance in wheat and understand the underlying molecular genetic mechanisms of root architecture and drought tolerance.
\end{abstract}

Keywords: Alien substitution lines, Drought, Individual seed weight, Root angle, Root length, Seed set percentage, Wheat

\footnotetext{
* Correspondence: vara@ksu.edu

1 Department of Agronomy, Kansas State University, Manhattan, Kansas 66506, USA

Full list of author information is available at the end of the article
}

(c) The Author(s). 2019 Open Access This article is distributed under the terms of the Creative Commons Attribution 4.0 International License (http://creativecommons.org/licenses/by/4.0/), which permits unrestricted use, distribution, and reproduction in any medium, provided you give appropriate credit to the original author(s) and the source, provide a link to the Creative Commons license, and indicate if changes were made. The Creative Commons Public Domain Dedication waiver (http://creativecommons.org/publicdomain/zero/1.0/) applies to the data made available in this article, unless otherwise stated. 


\section{Background}

Wheat (Triticum aestivum L.) is a major staple crop, and its production needs to increase by $\sim 38 \%$ by 2050 to feed the growing population [1]. Among the environmental changes expected in the future, climate models predict an increase in the variability of precipitation leading to an increased frequency and intensity of drought across the globe [2]. Globally, $\sim 79 \%$ of wheat harvesting regions show grain yield variability due to changes in precipitation and temperature [3]. Therefore, the enhancement of wheat drought tolerance (i.e., maintenance of high yields) is a key challenge in wheat improvement programs [4].

Bread wheat is an allohexaploid species (AABBDD genomes) that arose $\sim 8000$ years ago [5], from spontaneous hybridization of the tetraploid wheat $T$. turgidum L. (AABB genomes) with the diploid goatgrass Aegilops tauschii Coss. (DD genomes) [6,7]. Wheat has several primary, secondary and tertiary relatives spread across several genera including Triticum, Aegilops (Ae.), Dasypyrum, and Thinopyrum/Agropyron. Wild relatives of wheat are native to semi-arid zones of West and Central Asia and are therefore well adapted to various abiotic stresses $[8,9]$. Studies have shown that the introduction of alien chromosome segments from wild relatives into wheat have increased tolerance or resistance to drought [10], high temperatures [11], salinity [12], and water-logging [13]. Similarly, introduction of alien chromosome segments from wild relatives into wheat have improved pest resistance and yield [14, 15]. Waines and Ehdaie [15] and Yediay et al. [16] have sucessufully introgressed the genes associated with stem rust (Puccinia graminis) and powdery mildew (Blumeria graminis $\mathrm{f}$. sp. tritici) from rye (Secale cereale L.) to wheat germplasm, which resulted in increased stem rust and powdery mildew tolerance. The wheat lines showing rye-wheat translocation (1RS) had a positive performance on yield, root morphology, and water and nitrogen use efficiency [10]. Sequencing, expression studies, functional annotations, and high-throughput genomics analysis can accelerate allele mining for several traits in sets of chromosome segment substitution lines. Though genetic diversity in wild wheat is a useful resource for trait discovery, only limited numbers of wild relatives have been exploited due to the weedy morphology and low fertility of interspecific hybrids [17]. To utilize the genes and alleles conferring abiotic and biotic stress tolerance from wild relatives of wheat, different genetic materials in the form of addition, substitution, and translocation lines have been developed after laborious efforts in the last few decades [18]. Some translocation lines of wheat-Agropyron elongatum and wheat-rye have been studied for their response to drought stress [4, 10, 19], and lines with rye translocation $1 \mathrm{BL}-1 \mathrm{RS}$ have been used in cultivars across the world to enhance drought tolerance.

Roots appear to be the most relevant organ for breeding drought tolerance, yet limited research is available on root traits due to the difficulty of phenotyping and measuring under both field and controlled environments. Root system architecture refers to the spatial and temporal configuration of roots in the soil. Understanding the variability and contribution of specific root traits can help in the development of drought-tolerant genotypes. In most crops, genetic variation for root traits has been reported using mapping populations or wild relatives $[20,21]$. To our knowledge, genetic variation for root architecture in the alien chromosome addition/substitution or translocation lines involving Aegilops species and/or D. villosum under control and/or drought stress is not well understood. Placido et al. [4] reported that Ag. elongatum 7DL.7EL translocations in wheat improved seed yield under water limiting conditions by increasing root biomass. Similarly, a rye-wheat centric chromosome translocation 1RS.1BL increased yield under drought by enhancing root biomass [10, 19]. The yield advantage of the 1RS translocation line under water-limited conditions was partly associated with deceased root diameter, increased root length density and biomass [10]. Lukaszewski [22] observed three centric translocations, namely 1RS.1AL, 1RS.1BL, and 1RS.1DL, in Pavon 76 wheat background with greater root biomass and higher grain yield under irrigated and drought conditions. The genetic analyses of 1RS.1BS recombinant breakpoints in Pavon 76 indicated that the distal $15 \%$ of the physical length of chromosome 1RS might carry the gene(s) for better rooting ability and root morphological traits [19].

Relatively large root systems under drought can increase water uptake to alleviate the drought stress effect [23]. In contrast, since roots are a major sink for assimilates, reducing root biomass can increase the availability of assimilates for aboveground parts including grain [24]. Wheat production in India, Australia, and the United States represents a cross-section of global spring wheat production. In India, wheat is grown during winter (in the post-rainy season) and hence, is dependent on water stored in deep soil layers. Similarly, in north eastern Australia and the United States, spring wheat relies largely on stored soil moisture [25]. Hence, targeting access to deeper soil moisture with selected root traits in wheat is critical. Several root morphological traits like root angle, root diameter, and root length density have been associated with increased root system depth and water uptake [25-27]. Research into the physiological basis of drought tolerance in wheat is well established; however, the contribution of alien chromosome 
addition/substitution or translocation lines to drought tolerance is not fully understood. Osipova et al. [28] mapped quantitative trait locus (QTL) underlying chlorophyll fluorescence parameters and antioxidant enzyme on chromosome 7D of wheat under drought. Bobo et al. [29] reported that a spring wheat substitution line with the 3D chromosome from winter wheat had reduced quantum yield of photosystem II (ФPSII) under low light intensities. The substitution lines of durumChinese Spring (1B with $1 \mathrm{D}$ and $3 \mathrm{~B}$ with $3 \mathrm{D}$ chromosomes) had increased photosynthetic rates compared to the check, indicating that the $\mathrm{D}$ genome had a positive interaction with photosynthetic rate [30]. However, Haour-Lurton and Planchon [31] identified inhibitory effects of specific chromosomes of the $\mathrm{D}$ genome on photosynthesis. Wheat-barley addition lines $(7 \mathrm{H}$ and 7HL) had higher ФPSII, stomatal conductance and photosynthetic rates under salinity stress [32]. In another study, under terminal high temperature stress, the substitution lines of Chinese Spring (CS)-D. villosum (4 V.3 L, $6 \mathrm{~V} .3 \mathrm{~L}$, and 5S.3L) had increased chlorophyll index, ФPSII, individual seed weight and seed yield per plant than the check namely Chinese Spring [11]. Drought during gametogenesis causes a maximum reduction in grain number by inducing floret sterility in wheat and other crops [33]. However, drought during grain filling decreases individual grain weight $[33,34]$.

A combination of measuring and quantifying root phenotype and whole-plant physiological traits was used in this study to understand the drought-adaptive advantage introduced by the alien chromosome segment into wheat that can benefit wheat breeding programs focused on enhancing drought tolerance. The objectives of this research were to (i) quantify the effects of addition/substitution/translocation of chromosome segments from wheat wild relatives on the root, physiological and yield traits of hexaploid wheat under drought; and (ii) understand the mechanism(s) associated with drought tolerance or susceptibility in wheat-alien chromosome lines.

\section{Results}

\section{Principal component analyses}

The first two principal components (PC) from the principal component analysis (PCA) represented $54 \%$ of the variability, with $\mathrm{PC} 1$ explaining $40 \%$ of the variance. For $\mathrm{PC} 1$, the major contributors to the variance were seed yield per plant $(13 \%)$ and total root length (12\%). For $\mathrm{PC} 2$, the major contributors were maximum root length:shoot length ratio (28\%) and maximum root length (26\%) (Fig. 1a). The 48 wheat-alien chromosome lines and the CS check were grouped into four (I to IV) groups based on the PCA (Fig. 1b). The lines in group I (TA5088 and TA5638) and II (e.g., TA7619 and TA7598) were classified as tolerant and moderately tolerant,

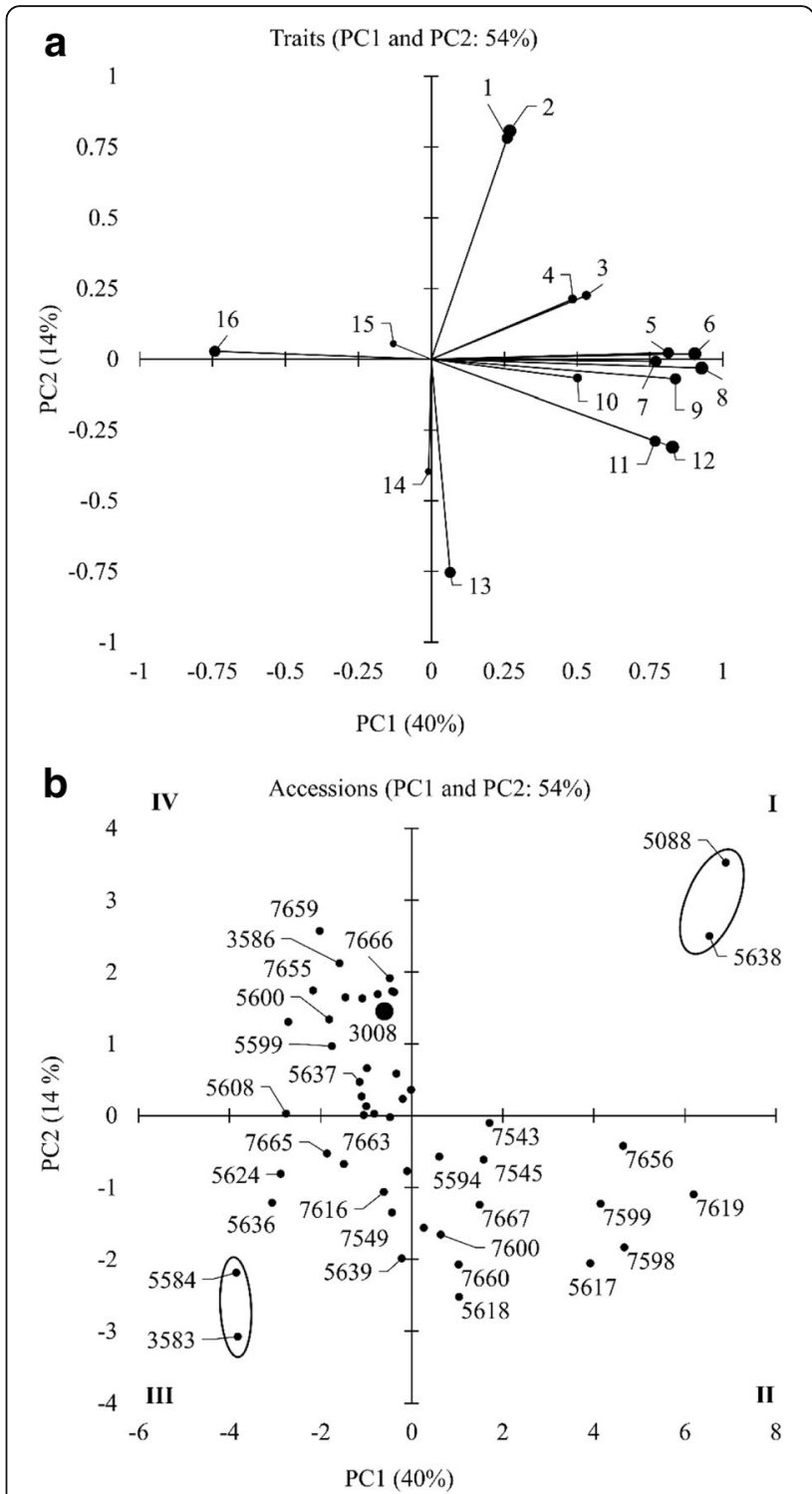

Fig. 1 Principal component analysis of chromosome substitution lines for identification of traits governing drought tolerance and genotypes with drought tolerance. (a) The principal component scores (PC1 and PC2) for identification of traits governing drought tolerance; (b) classification of 48 wheat-alien chromosome lines and the CS check based on factor scores of PC1 and PC2 for 16 trait variables. Description for (a) factor loading values for variables are indicated by black arrows radiating from the centre showing the direction (angle) and magnitude (length) and the contribution of the variables shown as the size of the circle, (b) four distinct groups are formed among the lines and the CS check (white circle).

Legends for (a) 1: maximum root length (cm); 2; maximum root length: shoot length ratio (unitless); 3 : number of seeds per plant, 4; number of seminal roots; 5 : seed set percentage, 6 : total root length (cm); 7: quantum yield of PSII (DPSII; unitless); 8: seed yield ( $\mathrm{g}$ plant $^{-1}$ ); 9: harvest index (\%); 10: chlorophyll index (SPAD units);11: root volume $\left(\mathrm{cm}^{3}\right)$; 12: individual seed weight $(\mathrm{mg})$; 13: seminal root angle $\left({ }^{\circ}\right)$; 14 : root diameter $(\mathrm{mm})$; 15 : seedling root length $(\mathrm{cm})$,i and 16: thylakoid membrane damage ( $F_{o} / F_{m}$ ratio; unitless). (b) I, II, III and IV indicates four groups. 3008, Chinese Spring 
respectively, to drought (Fig. 1b). The lines in group III (e.g., TA3583 and TA5584) and IV (CS and TA7659) were classified as susceptible and moderately susceptible, respectively to drought. Among the lines, the highest variation along PC1 was caused by TA5088 (7\%) followed by TA5638 (6.5\%), and in PC2 the highest variation was caused by TA5088 (3.5\%) followed by TA7659 (2.5\%) (Fig. 1b). The representative tolerant (TA5088 and TA5638) and susceptible genotypes (TA3583 and TA5584) for different traits were compared with a background check (CS) for root, physiological and yield traits to understand the mechanism of tolerance or susceptibility.

\section{Experiment 1. Genetic variability in seedling root traits}

The size of a plant's root system is a key trait that can affect the uptake of water from the soil. The 48 lines had significant $(P>0.001)$ genetic variability for the number of seminal roots (2.6-6.3), seedling root length (6.8$23.7 \mathrm{~cm})$ and root angle $\left(38.6-59.3^{\circ}\right)$; their corresponding mean values were $4.2,18.1 \mathrm{~cm}$ and $48.7^{\circ}$ (Table 1 ). Among the accessions, TA5088 and TA5638 had more seminal roots (6.3 vs. 2.6) and a lower root angle (av. 39 vs. $46^{\circ}$ ) than the CS check (Table 2). Accessions TA3583 and TA5584 had longer seedling roots (av. 22 vs. 10.9 $\mathrm{cm}$ ) and a greater root angle (59 vs. $46^{\circ}$ ) than the CS check but no significant difference in the number of seminal roots (Table 2).

\section{Experiment 2. Genetic variability in shoot and root traits under drought}

There were significant $(P>0.001)$ effects of genotype, drought, and their interaction for all growth and root-related traits; except the genotype $\times$ drought interactions for plant height and shoot dry weight per plant. Under drought, maximum root length ranged from $58.5-164.5 \mathrm{~cm}$, total root length ranged from 1826 to $10,873 \mathrm{~cm}$, maximum root length:shoot length ratio ranged from $0.95-2.90$ and root volume ranged from $0.55-4.60 \mathrm{~cm}^{3}$ in the 48 substitution lines (Table 3). Averaged across the lines, drought significantly $(P>0.001)$ reduced plant height (7\%), maximum root length (26\%), maximum root length:shoot length ratio $(21 \%)$, total root length (7\%), root diameter (12\%), root volume

Table 1 Range, mean and least significant difference (LSD) for the number of seminal roots, seedling root length $(\mathrm{cm})$, and root angle $\left(^{\circ}\right)$ among the wheat--alien chromosome lines (Experiment 1)

\begin{tabular}{llll}
\hline Trait & Range & Mean & LSD \\
\hline Number of seminal roots & $2.6-6.3$ & 4.2 & $1.1^{* * *}$ \\
Seedling root length $(\mathrm{cm})$ & $6.8-23.7$ & 18.1 & $2.5^{* * *}$ \\
Root angle $\left(^{\circ}\right)$ & $38.6-59.3$ & 48.7 & $6.3^{* * *}$ \\
\hline *** indicates $P \leq 0.001$ & & &
\end{tabular}

(23\%), number of tillers per plant (33\%), and stem dry weight per plant (42\%), relative to the control indicating that both shoot and root growth are affected by drought stress (Table 3).

Accessions TA5088 and TA5638 had significantly $(P>$ 0.001 ) higher maximum root length (av. $161 \mathrm{vs.} 124 \mathrm{~cm}$ ), maximum root length:shoot length ratio (av. 2.8 vs. 2.2) and total root length (av. 10,517 vs. $3768 \mathrm{~cm}$ ) than the CS check under drought (Table 4). However, the lines TA3583 and TA5584 had lower maximum root length (av. $73 \mathrm{vs.} 124 \mathrm{~cm}$ ), maximum root length:root length ratio (av. 1.2 vs. 2.2) and total root length (av. 2459 vs. $3768 \mathrm{~cm}$ ) than the CS check under drought (Table 4). Compared to CS, higher maximum root length and total root length was observed in the accessions TA5088 and TA5638 due to its narrow root angle.

\section{Experiment 3. Genetic variability in physiological and yield traits under drought}

There were significant $(P>0.001)$ effects of genotype, drought, and their interaction for chlorophyll index (SPAD units), thylakoid membrane damage $\left(\mathrm{F}_{\mathrm{o}} / \mathrm{F}_{\mathrm{m}}\right.$ ratio; unitless), ФPSII (unitless), electron transport rate ( $\mu \mathrm{mol}$ electrons $\mathrm{m}^{-2} \mathrm{~s}^{-1}$ ), seed set percentage, seed yield ( $\mathrm{g}$ spike ${ }^{-1}$ ), seed number plant $^{-1}$, individual seed weight $\left(\mathrm{mg} \mathrm{seed}^{-1}\right)$, seed yield $\left(\mathrm{g} \mathrm{plant}^{-1}\right)$ and harvest index (\%) (Table 5). There was wide genetic variability for various physiological and yield traits under control and drought conditions. Among the 48 lines, thylakoid membrane damage and the ФPSII ranged from $0.255-0.425$, and 0.105-0.465, respectively, under drought. Similarly, seed set percentage and number of seeds per plant ranged between $6.7-59.8 \%$ and $2.0-89.0$ plant $^{-1}$, respectively. Individual seed weights ranged from $3.9-59.9 \mathrm{mg}$ with a mean of $22.8 \mathrm{mg}$ under drought. Seed yield and harvest index ranged between $0.02-2.0 \mathrm{~g} \mathrm{plant}^{-1}$ and from 0.18 $29.5 \%$, respectively, under drought. Irrespective of the line, drought significantly $(P>0.001)$ reduced chlorophyll index (26\%), ФPSII (43\%), electron transport rate (44\%), seed set percentage (52\%), seed yield per spike (63\%), number of seeds (60\%), individual seed weight (23\%), seed yield per plant (66\%), and harvest index (54\%) but increased thylakoid membrane damage (67\%) compared to the control. Overall, the result indicates drought stress had negative effect on both photosynthetic efficiency and yield associated traits. Comparing both photosynthetic efficiency and yield traits the later was found to be more sensitive than former.

The ranking of wheat-alien chromosome lines based on the numerical values of different physiological traits are in Table 6. The data indicate that drought stress increases damage to the thylakoid membrane, and reduces chlorophyll content and ФPSII in both the deep and shallow rooting accessions, however, the damage was 
Table 2 Mean values for the number of seminal roots, seedling root length (cm) and root angle $\left(^{\circ}\right)$ of the six highest (top) and six lowest (bottom) ranked wheat-alien chromosome lines along with the CS check (Experiment 1)

\begin{tabular}{|c|c|c|c|}
\hline Rank & Number of seminal roots & Seedling root length $(\mathrm{cm})$ & Root angle $\left(^{\circ}\right)$ \\
\hline \multicolumn{4}{|l|}{ Top 6} \\
\hline & TA5088 $(6.3 \pm 0.52)$ & TA5636 (23.0 \pm 0.55$)$ & TA7660 $(59.3 \pm 1.20)$ \\
\hline & TA5638 $(6.3 \pm 0.51)$ & TA5584 $(22.6 \pm 0.79)$ & TA3583 $(59.0 \pm 0.33)$ \\
\hline & TA7667 $(6.3 \pm 0.67)$ & TA7655 $(22.0 \pm 0.10)$ & TA5584 $(59.0 \pm 1.53)$ \\
\hline & TA7599 $(6.0 \pm 0.11)$ & TA7598 $(22.0 \pm 0.29)$ & TA7598 $(57.0 \pm 3.48)$ \\
\hline & TA7619 (5.0 \pm 0.58$)$ & TA3583 $(21.6 \pm 0.26)$ & TA5594 $(56.0 \pm 2.31)$ \\
\hline & TA7688 (5.0 \pm 0.58$)$ & TA6656 (21.5 \pm 0.88$)$ & TA5618 $(55.0 \pm 1.53)$ \\
\hline \multicolumn{4}{|c|}{ Bottom 6} \\
\hline & TA3583 $(3.3 \pm 0.33)$ & TA7660 $(13.4 \pm 0.14)$ & TA7657 $(41.3 \pm 0.33)$ \\
\hline & TA5584 $(3.3 \pm 0.33)$ & TA7616 $(12.1 \pm 0.27)$ & TA7544 $(41.0 \pm 2.41)$ \\
\hline & TA5637 (3.3 \pm 0.33$)$ & TA5616 (12.0 \pm 0.13$)$ & TA5624 (40.7 \pm 0.58$)$ \\
\hline & TA7543 $(3.3 \pm 0.33)$ & TA7620 $(11.7 \pm 0.11)$ & TA5638 $(40.0 \pm 2.67)$ \\
\hline & TA7547 (3.3 \pm 0.33$)$ & TA7667 $(10.1 \pm 0.50)$ & TA6656 (38.7 \pm 1.86$)$ \\
\hline & TA5657 $(2.6 \pm 0.33)$ & TA7549 $(6.8 \pm 0.11)$ & TA5088 $(38.3 \pm 2.66)$ \\
\hline \multicolumn{4}{|c|}{ Check (CS) } \\
\hline & $3008(2.6 \pm 0.33)$ & $3008(10.9 \pm 0.58)$ & $3008(46.0 \pm 0.58)$ \\
\hline LSD & 1.1 & 2.5 & 6.3 \\
\hline
\end{tabular}

Genotypes were ranked based on numerical values. Values in parentheses are means \pm standard error of the respective trait. CS Chinese Spring, LSD least significant difference

lower in the deep rooting accessions than in the shallow rooting accessions. The lines TA5088 and TA5638 had a higher chlorophyll index and ФPSII and lower thylakoid membrane damage than the CS check under drought (Table 6), and higher seed set percentages (55.8 and $59.8 \%$, respectively), individual seed weights (44.5 and $45.5 \mathrm{mg} \mathrm{seed}^{-1}$, respectively) and seed yields (2.27 and $1.61 \mathrm{~g} \mathrm{plant}^{-1}$, respectively) than the CS check (34.0\%, $13.2 \mathrm{mg} \mathrm{seed}^{-1}$ and $0.51 \mathrm{~g} \mathrm{plant}^{-1}$, respectively); both lines were grouped with the six best-performing genotypes under drought (Table 7). The lines TA3583 and
TA5584 had lower seed set percentages (15.9 and 15.7\%, respectively), individual seed weights $(9.4$ and $7.8 \mathrm{mg}$ seed $^{-1}$, respectively), and seed yields $(0.07$ and $0.07 \mathrm{~g}$ plant $^{-1}$, respectively) than the CS check under drought. The higher seed set percentage and individual seed weight was observed in the accessions TA5088 and TA5638 compared to CS, resulting in higher seed yields.

\section{Relationship among root, physiological and yield traits}

Total root length was positively associated with chlorophyll index $\left(r^{2}=0.21\right.$; Fig. 2a), ФPSII $\left(r^{2}=0.39\right.$; Fig. 2 c $)$,

Table 3 Range, mean and least significant difference (LSD) for plant height (cm), maximum root length (rooting depth; $\mathrm{cm}$ ), maximum root length:shoot length ratio (unitless), total root length $(\mathrm{cm})$, root diameter $(\mathrm{mm})$, root volume $\left(\mathrm{cm}^{3}\right)$, tiller number (plant ${ }^{-1}$ ) and stem dry weight ( plant $^{-1}$ ) of wheat-alien chromosome lines under irrigation (control) and drought stress (water withheld for 58 d) (Experiment 2)

\begin{tabular}{|c|c|c|c|c|c|}
\hline \multirow[t]{2}{*}{ Trait } & \multicolumn{2}{|c|}{ Irrigated condition } & \multicolumn{2}{|c|}{ Drought stress } & \multirow{2}{*}{$\begin{array}{l}\text { LSD for } \\
\text { stress }\end{array}$} \\
\hline & Range & Mean & Range & $\overline{\text { Mean }}$ & \\
\hline Plant height $(\mathrm{cm})$ & $44.0-102.5$ & 63.4 & $39.6-97.0$ & 58.7 & $2.8^{* * * *}$ \\
\hline Maximum root length (rooting depth; $\mathrm{cm}$ ) & $108.0-186.0$ & 146.9 & $58.5-164.5$ & 108.7 & $5.8^{* * *}$ \\
\hline Maximum root length:shoot length ratio (unitless) & $1.15-3.20$ & 2.39 & $0.95-2.90$ & 1.90 & $0.11^{* * *}$ \\
\hline Total root length $(\mathrm{cm})$ & $1559-11,599$ & 5131 & $1826-10,873$ & 4747 & $276^{* * *}$ \\
\hline Root diameter (mm) & $0.19-0.32$ & 0.26 & $0.17-0.29$ & 0.23 & $0.009^{* * *}$ \\
\hline Root volume $\left(\mathrm{cm}^{3}\right)$ & $0.70-5.50$ & 2.6 & $0.55-4.60$ & 2.0 & $0.2^{* * *}$ \\
\hline Tiller number $\left(\right.$ plant $^{-1}$ ) & $3.5-12.5$ & 5.8 & $1.5-12.0$ & 3.9 & $0.45^{* * *}$ \\
\hline Stem dry weight (g plant ${ }^{-1}$ ) & $0.41-6.40$ & 2.6 & $0.28-4.70$ & 1.5 & $0.25^{* * *}$ \\
\hline
\end{tabular}

*** indicates $P \leq 0.001$ 
Table 4 Mean values for maximum root length (rooting depth; $\mathrm{cm}$ ), maximum root length:shoot length ratio (unitless) and total root length (cm) of the six highest (top) and six lowest (bottom) ranked wheat-alien chromosome lines along with the CS check under irrigation (control) and drought stress (water withheld for $58 \mathrm{~d}$ ) (Experiment 2)

\begin{tabular}{|c|c|c|c|c|c|c|c|c|c|}
\hline \multirow[t]{2}{*}{ Rank } & \multicolumn{3}{|c|}{ Maximum root length $(\mathrm{cm})$} & \multicolumn{3}{|c|}{$\begin{array}{l}\text { Maximum root length:shoot length ratio } \\
\text { (unitless) }\end{array}$} & \multicolumn{3}{|c|}{ Total root length $(\mathrm{cm})$} \\
\hline & Line & Irrigated & Drought & Line & Irrigated & Drought & Line & Irrigated & Drought \\
\hline \multicolumn{10}{|l|}{ Top 6} \\
\hline & TA7659 & $155.0 \pm 3.2$ & $164.5 \pm 4.2$ & TA5088 & $3.10 \pm 0.05$ & $2.90 \pm 0.11$ & TA5638 & $7643 \pm 487$ & $10,873 \pm 44$ \\
\hline & TA5088 & $171.5 \pm 6.5$ & $161.5 \pm 4.2$ & TA5638 & $2.35 \pm 0.04$ & $2.70 \pm 0.06$ & TA5088 & $11,600 \pm 2329$ & $10,162 \pm 51$ \\
\hline & TA5638 & $152.0 \pm 7.0$ & $161.0 \pm 7.0$ & TA3586 & $3.15 \pm 0.05$ & $2.65 \pm 0.41$ & TA7598 & $4325 \pm 804$ & $8643 \pm 1312$ \\
\hline & TA7662 & $116.0 \pm 4.0$ & $145.0 \pm 14.1$ & TA7594 & $2.60 \pm 0.04$ & $2.55 \pm 0.42$ & TA7656 & $7235 \pm 184$ & $8511 \pm 590$ \\
\hline & TA7657 & $114.5 \pm 14.5$ & $135.0 \pm 7.8$ & TA7655 & $2.10 \pm 0.05$ & $2.55 \pm 0.11$ & TA7619 & $3544 \pm 66$ & $8423 \pm 86$ \\
\hline & TA7663 & $109.5 \pm 11.5$ & $133.0 \pm 14.3$ & TA3585 & $3.15 \pm 0.35$ & $2.50 \pm 0.14$ & TA7545 & $6042 \pm 566$ & $7840 \pm 68$ \\
\hline \multicolumn{10}{|l|}{ Bottom 6} \\
\hline & TA5584 & $174.5 \pm 2.4$ & $75.5 \pm 2.9$ & TA5584 & $2.75 \pm 0.19$ & $1.40 \pm 0.20$ & TA3586 & $3124 \pm 1608$ & $2500 \pm 308$ \\
\hline & TA5618 & $168.0 \pm 2.0$ & $75.5 \pm 10.3$ & TA7663 & $1.25 \pm 0.11$ & $1.35 \pm 0.35$ & TA5584 & $3634 \pm 530$ & $2491 \pm 135$ \\
\hline & TA7600 & $137.0 \pm 2.0$ & $75.5 \pm 8.3$ & TA5636 & $2.41 \pm 0.19$ & $1.30 \pm 0.20$ & TA3583 & $5619 \pm 568$ & $2427 \pm 89$ \\
\hline & TA7620 & $149.5 \pm 23.8$ & $74.0 \pm 16.7$ & TA3583 & $2.60 \pm 0.29$ & $1.10 \pm 0.40$ & TA5636 & $2873 \pm 1408$ & $2378 \pm 39$ \\
\hline & TA3583 & $159.0 \pm 23.8$ & $71.0 \pm 5.7$ & TA7620 & $2.25 \pm 0.37$ & $1.05 \pm 0.15$ & TA7616 & $4385 \pm 1324$ & $2198 \pm 105$ \\
\hline & TA5639 & $152.50 \pm 2.4$ & $58.5 \pm 20.1$ & TA5639 & $2.21 \pm 0.11$ & $0.95 \pm 0.45$ & TA5599 & $6147 \pm 412$ & $1826 \pm 258$ \\
\hline \multicolumn{10}{|l|}{ Check (CS) } \\
\hline & TA3008 & $151.0 \pm 24.5$ & $124.0 \pm 4.9$ & TA3008 & $2.36 \pm 0.15$ & $2.20 \pm 0.16$ & TA3008 & $4195 \pm 336$ & $3768 \pm 822$ \\
\hline LSD (Genotype) & & & 28.8 & & & 0.55 & & & 1368 \\
\hline LSD (Genotype x Stress) & & & 5.8 & & & 0.11 & & & 276 \\
\hline
\end{tabular}

Genotypes were ranked based on the numerical values under drought stress. Values are means \pm standard error of the respective trait. CS Chinese Spring, LSD least significant difference

Table 5 Range, mean and least significant difference (LSD) for chlorophyll index (SPAD units), thylakoid membrane damage ( $F_{0} / F_{m}$ ratio; unitless), quantum yield of PSII (DPSIl; unitless), electron transport rate ( $\mu$ mol electrons $\mathrm{m}^{-2} \mathrm{~s}^{-1}$ ), seed set percentage, seed yield $\left(\mathrm{g} \mathrm{spike}^{-1}\right)$, seed number (plant $\left.{ }^{-1}\right)$, individual seed weight $\left(\mathrm{mg} \mathrm{seed}^{-1}\right)$, seed yield $\left(\mathrm{g} \mathrm{plant}^{-1}\right)$ and harvest index $(\%)$ of wheatalien chromosome lines under irrigation (control) and drought stress (water withheld for $16 \mathrm{~d}$ ) (Experiment 3). ${ }^{* * *}$ indicates $P \leq 0.001$

\begin{tabular}{|c|c|c|c|c|c|}
\hline \multirow[t]{2}{*}{ Trait } & \multicolumn{2}{|c|}{ Irrigated condition } & \multicolumn{2}{|c|}{ Drought stress } & \multirow{2}{*}{$\begin{array}{l}\text { LSD for } \\
\text { stress }\end{array}$} \\
\hline & Range & Mean & Range & Mean & \\
\hline Chlorophyll index (SPAD units) & $30.0-54.2$ & 41.8 & $19.9-49.3$ & 31.1 & $0.38^{* * *}$ \\
\hline Thylakoid membrane damage ( $F_{o} / F_{m}$ ratio; unitless) & $0.140-0.260$ & 0.203 & $0.255-0.425$ & 0.340 & $0.003^{* * *}$ \\
\hline Quantum yield of PSII (DPSII; unitless) & $0.345-0.590$ & 0.462 & $0.105-0.465$ & 0.262 & $0.005^{* * *}$ \\
\hline Electron transport rate ( $\mu \mathrm{mol}$ electrons $\mathrm{m}^{-2} \mathrm{~s}^{-1}$ ) & $197.3-297.6$ & 242.2 & $50.6-215.3$ & 135.8 & $3.0^{* * *}$ \\
\hline Seed set percentage & $44.5-84.0$ & 68.0 & $6.7-59.8$ & 32.9 & $0.84^{* * *}$ \\
\hline Seed yield (g spike ${ }^{-1}$ ) & $0.12-1.91$ & 0.92 & $0.02-1.26$ & 0.34 & $0.02^{* * *}$ \\
\hline Seed number $\left(\right.$ plant $\left.^{-1}\right)$ & $23.5-133.2$ & 57.1 & $2.0-89.0$ & 23.0 & $2.1^{* * *}$ \\
\hline Individual seed weight $\left(\mathrm{mg} \mathrm{seed}^{-1}\right.$ ) & $6.0-56.0$ & 29.6 & $3.9-59.9$ & 22.8 & $1.3^{* * *}$ \\
\hline Seed yield (g plant ${ }^{-1}$ ) & $0.17-3.5$ & 1.6 & $0.02-2.0$ & 0.54 & $0.04^{* * *}$ \\
\hline Harvest index (\%) & $2.5-31.3$ & 15.2 & $0.18-29.5$ & 7.0 & $0.44^{* * *}$ \\
\hline
\end{tabular}

*** indicates $P \leq 0.001$ 
Table 6 Mean values for chlorophyll index (SPAD units), thylakoid membrane damage $\left(F_{0} / F_{m}\right.$ ratio; unitless) and quantum yield of PSII (DPSII; unitless) of six highest (top) and six lowest (bottom) ranked wheat-alien chromosome lines along with CS check under irrigation (control) and drought stress (water withheld for $16 \mathrm{~d}$ ) (Experiment 3)

\begin{tabular}{|c|c|c|c|c|c|c|c|c|c|}
\hline \multirow[t]{2}{*}{ Rank } & \multicolumn{3}{|c|}{ Chlorophyll index (SPAD units) } & \multicolumn{3}{|c|}{$\begin{array}{l}\text { Thylakoid membrane damage ( } F_{o} / F_{m} \text { ratio; } \\
\text { unitless) }\end{array}$} & \multicolumn{3}{|c|}{$\begin{array}{l}\text { Quantum yield of PSII (DPSII; } \\
\text { unitless) }\end{array}$} \\
\hline & Line & Irrigated & Drought & Line & Irrigated & Drought & Line & Irrigated & Drought \\
\hline \multicolumn{10}{|l|}{ Top 6} \\
\hline & TA7598 & $54.25 \pm 0.65$ & $49.30 \pm 0.80$ & TA5608 & $0.200 \pm 0.012$ & $0.425 \pm 0.012$ & TA7656 & $0.58 \pm 0.02$ & $0.47 \pm 0.03$ \\
\hline & TA7600 & $40.30 \pm 1.20$ & $43.15 \pm 1.45$ & TA7659 & $0.195 \pm 0.011$ & $0.420 \pm 0.021$ & TA7619 & $0.53 \pm 0.01$ & $0.45 \pm 0.02$ \\
\hline & TA7543 & $42.10 \pm 0.50$ & $38.25 \pm 0.15$ & TA5599 & $0.215 \pm 0.011$ & $0.415 \pm 0.011$ & TA7543 & $0.49 \pm 0.01$ & $0.43 \pm 0.02$ \\
\hline & TA7655 & $42.30 \pm 0.40$ & $38.15 \pm 0.45$ & TA5600 & $0.185 \pm 0.012$ & $0.405 \pm 0.012$ & TA5088 & $0.59 \pm 0.01$ & $0.40 \pm 0.01$ \\
\hline & TA5638 & $47.15 \pm 1.65$ & $38.10 \pm 0.55$ & TA5584 & $0.195 \pm 0.013$ & $0.405 \pm 0.011$ & TA5638 & $0.51 \pm 0.01$ & $0.39 \pm 0.01$ \\
\hline & TA5088 & $46.15 \pm 0.35$ & $36.90 \pm 1.30$ & TA3583 & $0.225 \pm 0.011$ & $0.400 \pm 0.011$ & TA7545 & $0.47 \pm 0.01$ & $0.37 \pm 0.01$ \\
\hline \multicolumn{10}{|l|}{ Bottom 6} \\
\hline & TA5637 & $43.60 \pm 1.30$ & $23.80 \pm 0.45$ & TA7599 & $0.190 \pm 0.010$ & $0.260 \pm 0.012$ & TA7544 & $0.53 \pm 0.01$ & $0.13 \pm 0.01$ \\
\hline & TA3583 & $47.85 \pm 1.76$ & $21.45 \pm 1.15$ & TA5088 & $0.195 \pm 0.012$ & $0.255 \pm 0.016$ & TA7667 & $0.37 \pm 0.01$ & $0.13 \pm 0.01$ \\
\hline & TA7657 & $34.85 \pm 0.75$ & $20.85 \pm 0.75$ & TA5617 & $0.220 \pm 0.015$ & $0.255 \pm 0.013$ & TA6656 & $0.43 \pm 0.01$ & $0.12 \pm 0.01$ \\
\hline & TA5640 & $30.00 \pm 0.45$ & $20.75 \pm 0.35$ & TA5638 & $0.155 \pm 0.016$ & $0.255 \pm 0.012$ & TA3583 & $0.46 \pm 0.01$ & $0.11 \pm 0.01$ \\
\hline & TA7664 & $34.50 \pm 1.10$ & $19.95 \pm 0.36$ & TA7619 & $0.210 \pm 0.012$ & $0.255 \pm 0.012$ & TA5584 & $0.52 \pm 0.01$ & $0.11 \pm 0.01$ \\
\hline & TA5584 & $39.35 \pm 0.45$ & $19.60 \pm 1.02$ & TA7656 & $0.195 \pm 0.015$ & $0.255 \pm 0.011$ & TA5636 & $0.47 \pm 0.01$ & $0.11 \pm 0.01$ \\
\hline \multicolumn{10}{|l|}{ Check (CS) } \\
\hline & TA3008 & $32.3 \pm 1.10$ & $21.05 \pm 0.45$ & TA3008 & $0.220 \pm 0.011$ & $0.355 \pm 0.011$ & TA3008 & $0.47 \pm 0.03$ & $0.32 \pm 0.01$ \\
\hline LSD (Genotype) & & & 1.89 & & & 0.017 & & & 0.020 \\
\hline LSD (Genotype x Stress) & & & 1.86 & & & 0.003 & & & 0.005 \\
\hline
\end{tabular}

Genotypes were ranked based on numerical values under drought stress. Values are means \pm standard error of the respective trait. CS Chinese Spring, LSD least significant difference

electron transport rate $\left(r^{2}=0.60\right.$; Fig. $\left.2 \mathrm{~d}\right)$, seed set percentage $\left(r^{2}=0.52\right.$; Fig. $\left.2 \mathrm{e}\right)$, number of seeds per plant $\left(r^{2}=0.16\right.$; Fig. 2f), individual seed weight $\left(r^{2}=0.35\right.$; Fig. $2 \mathrm{~g})$ and seed yield per plant $\left(r^{2}=0.54\right.$; Fig. $\left.2 \mathrm{~h}\right)$ in the wheat-alien chromosome lines under drought. However, thylakoid membrane damage had a negative association with total root length $\left(r^{2}=0.39\right.$; Fig. $\left.2 \mathrm{~b}\right)$. The lines with more total root length had higher seed set percentage, individual seed weights and seed yields per plant under drought (Fig. 2e, g, h). Although all the above-mentioned traits were associated with total root length, the association between total root length and electron transport rate and seed yield per plant was higher than other traits indicating that these traits might be physiologically related.

The ФPSII had a positive relationship [co-efficient of determination $\left(r^{2}\right) \geq 0.39$ ] with seed set percentage, individual seed weight, seed yield per plant and harvest index (Fig. 3c-f) but a negative relationship with thylakoid membrane damage $\left(r^{2}=0.33\right.$; Fig. $\left.3 b\right)$ under drought in the wheat-alien chromosome lines. The lines with a higher ФPSII had higher seed set percentages, individual seed weights and seed yields per plant (Fig. 3c-e).

\section{Discussion}

This study demonstrated that (i) alien segments in the wheat background have altered root architecture and largely reduced photochemical efficiency and seed yield components under drought, (ii) accessions TA5088 and TA5638 with alien chromosome segments from Ae. speltoides (5S) and D. villosum (5 V) in CS wheat, respectively were drought tolerant, and (iii) the drought tolerance mechanism is associated with a deep, thin and profuse root system.

Root traits play a significant role in plants exposed to drought [35]. Plant capacity to extract soil moisture is a key factor determining drought adaptation, which is likely to result from the improved exploration of soil water [36]. PCA identified accessions TA5088 and TA5638 as drought tolerant (Fig. 1b) and accessions TA3583 and TA5584 as drought susceptible (Fig. 1b) based on 16 root, shoot and yield traits. Accession TA5088 has the long arm of chromosome 5S from Ae. speltoides and TA5638 have the short arm of chromosome $5 \mathrm{~V}$ from $D$. villosum. Ae. speltoides and D. villosum are wild relatives of wheat and rich sources of genetic variation for resistance to drought $[37,38]$. The effect of $5 \mathrm{~S}$ and $5 \mathrm{~V}$ translocation in bread wheat was 
Table 7 Mean values for seed set percentage, individual seed weight ( $\mathrm{mg} \mathrm{seed}^{-1}$ ) and seed yield ( $\mathrm{g} \mathrm{plant}{ }^{-1}$ ) of six highest (top) and six lowest (bottom) ranked wheat-alien chromosome lines along with CS check under irrigation (control) and drought stress (water withheld for $16 \mathrm{~d}$ ) (Experiment 3)

\begin{tabular}{|c|c|c|c|c|c|c|c|c|c|}
\hline \multirow[t]{2}{*}{ Rank } & \multicolumn{3}{|c|}{ Seed set percentage } & \multicolumn{3}{|c|}{ Individual seed weight $\left(\mathrm{mg} \mathrm{seed}^{-1}\right)$} & \multicolumn{3}{|c|}{ Seed yield $\left(\mathrm{g} \mathrm{plant}^{-1}\right.$ ) } \\
\hline & Line & Irrigated & Drought & Line & Irrigated & Drought & Line & Irrigated & Drought \\
\hline \multicolumn{10}{|l|}{ Top 6} \\
\hline & TA5638 & $82.4 \pm 1.9$ & $59.8 \pm 2.1$ & TA7598 & $37.1 \pm 1.8$ & $60.0 \pm 4.5$ & TA5088 & $3.42 \pm 0.13$ & $2.27 \pm 0.07$ \\
\hline & TA7545 & $69.1 \pm 2.9$ & $55.8 \pm 1.3$ & TA7619 & $43.6 \pm 2.2$ & $56.9 \pm 3.7$ & TA7619 & $2.49 \pm 0.14$ & $1.78 \pm 0.08$ \\
\hline & TA5088 & $68.5 \pm 1.5$ & $55.8 \pm 2.4$ & TA7599 & $56.0 \pm 3.4$ & $55.0 \pm 3.6$ & TA5638 & $2.19 \pm 0.10$ & $1.61 \pm 0.11$ \\
\hline & TA7619 & $66.4 \pm 1.1$ & $53.2 \pm 1.1$ & TA5617 & $42.7 \pm 3.8$ & $50.4 \pm 6.5$ & TA7598 & $1.88 \pm 0.13$ & $1.31 \pm 0.07$ \\
\hline & TA5617 & $66.5 \pm 2.1$ & $51.7 \pm 3.9$ & TA5638 & $22.9 \pm 1.0$ & $45.5 \pm 2.4$ & TA5617 & $1.63 \pm 0.30$ & $1.29 \pm 0.10$ \\
\hline & TA7656 & $63.8 \pm 1.2$ & $48.4 \pm 1.6$ & TA5088 & $45.0 \pm 1.6$ & $44.5 \pm 4.4$ & TA7599 & $1.69 \pm 0.10$ & $1.26 \pm 0.07$ \\
\hline \multicolumn{10}{|l|}{ Bottom 6} \\
\hline & TA3583 & $72.3 \pm 3.7$ & $15.9 \pm 1.5$ & TA3583 & $10.6 \pm 0.4$ & $9.4 \pm 1.1$ & TA7665 & $0.76 \pm 0.06$ & $0.11 \pm 0.01$ \\
\hline & TA5584 & $66.0 \pm 1.8$ & $15.7 \pm 1.0$ & TA7666 & $17.4 \pm 2.3$ & $9.2 \pm 2.2$ & TA5584 & $0.29 \pm 0.01$ & $0.07 \pm 0.01$ \\
\hline & TA7544 & $61.4 \pm 1.4$ & $14.9 \pm 1.2$ & TA7655 & $11.8 \pm 2.2$ & $8.8 \pm 1.3$ & TA3583 & $0.262 \pm 0.01$ & $0.07 \pm 0.01$ \\
\hline & TA5624 & $71.2 \pm 2.7$ & $13.4 \pm 1.1$ & TA5624 & $12.9 \pm 0.7$ & $8.6 \pm 0.6$ & TA5624 & $0.45 \pm 0.02$ & $0.06 \pm 0.02$ \\
\hline & TA7688 & $68.0 \pm 1.3$ & $12.8 \pm 1.1$ & TA5584 & $6.0 \pm 0.4$ & $7.8 \pm 0.8$ & TA7544 & $0.72 \pm 0.01$ & $0.04 \pm 0.01$ \\
\hline & TA7655 & $48.2 \pm 2.5$ & $6.7 \pm 1.2$ & TA7544 & $26.8 \pm 1.0$ & $6.7 \pm 1.7$ & TA7655 & $0.30 \pm 0.05$ & $0.02 \pm 0.01$ \\
\hline \multicolumn{10}{|l|}{ Check (CS) } \\
\hline & TA3008 & $68.53 \pm 4.0$ & $34.00 \pm 2.6$ & TA3008 & $21.65 \pm 1.2$ & $13.2 \pm 1.8$ & TA3008 & $2.46 \pm 0.14$ & $0.51 \pm 0.04$ \\
\hline LSD (Genotype) & & & 4.10 & & & 6.5 & & & 0.21 \\
\hline LSD (Genotype x Stress) & & & 0.84 & & & 1.3 & & & 0.04 \\
\hline
\end{tabular}

Genotypes were ranked based on the numerical values under drought stress. Values are means \pm standard error of the respective trait. CS Chinese Spring, LSD least significant difference. The top and bottom six genotypes are wheat-alien chromosome lines

evident from the rooting depth, total root length, and root diameter. In general, cereal roots do not have a vascular cambium and must develop more root length to generate new xylem tissues for water transport [39]. Hence, in water-limiting situations, the genotypes with increased root length had more opportunity for water uptake. This has been confirmed in rice, wheat and maize as deep rooting with profuse branching and small root diameters are associated with drought tolerance [25-27, 40]. In contrast, accession TA3583 with a monosomic addition for chromosome $4 \mathrm{~S}$ from Ae. searsii and TA5584 with the chromosome $7 \mathrm{~J}$ arm translocated from Th. Intermedium had wider root angles with sparse root branching at depth and increased root diameter, which is associated with topsoil foraging that eventually results in drought susceptibility [25]. Hence, it is postulated that the gene(s) associated with deep rooting and profuse branching are present in $5 \mathrm{~S}$ of Ae. speltoides and $5 \mathrm{~V}$ of $D$. villosum and the gene(s) associated with shallow rooting and sparse branching are localized at $4 \mathrm{~S}$ and $7 \mathrm{~J}$ of Ae. searsii and Th. intermedium, respectively.

Studying root architecture and associating it with crop performance under drought can help to identify proxy traits for improving drought tolerance in wheat $[36,40]$. The present study clearly showed that total root length and ФPSII could be used as a proxy trait to evaluate drought tolerance in wheat because of its strong, positive relationship between seed set percentage and seed yield per plant under drought (Fig. 3a). Under drought, the chlorophyll index, ФPSII and electron transport rate decreased in all 48 lines but the thylakoid membrane damage increased (Table 5). The structural and functional alterations in chloroplast under drought namely damages to thylakoid membranes may lead to chlorophyll loss because chlorophyll is primarily located in the thylakoid membranes [41]. The decreased ФPSII and electron transport rate under drought shows the occurrence of photoinhibition [42, 43]; and it could be associated with the generation of excess excitation energy [43]. The observed genetic variability for chlorophyll index and ФPSII in the wheat-alien chromosome lines falls within the range reported by Pour-Aboughadareh et al. [44]. Under drought stress, the ФPSII in TA5088 and TA5638 increased more (av. 25\%) than CS check. Chromosome substitution lines developed from synthetic wheat in the CS background showed that genes regulating $F_{\mathrm{o}}, \mathrm{F}_{\mathrm{m}}$ and ФPSII values might be located on the $3 \mathrm{~A}, 4 \mathrm{D}$ and $7 \mathrm{~A}$ chromosomes [45]. Higher chlorophyll content and lower membrane damage under drought stress in the tolerant genotype of wheat have been reported [46]. 


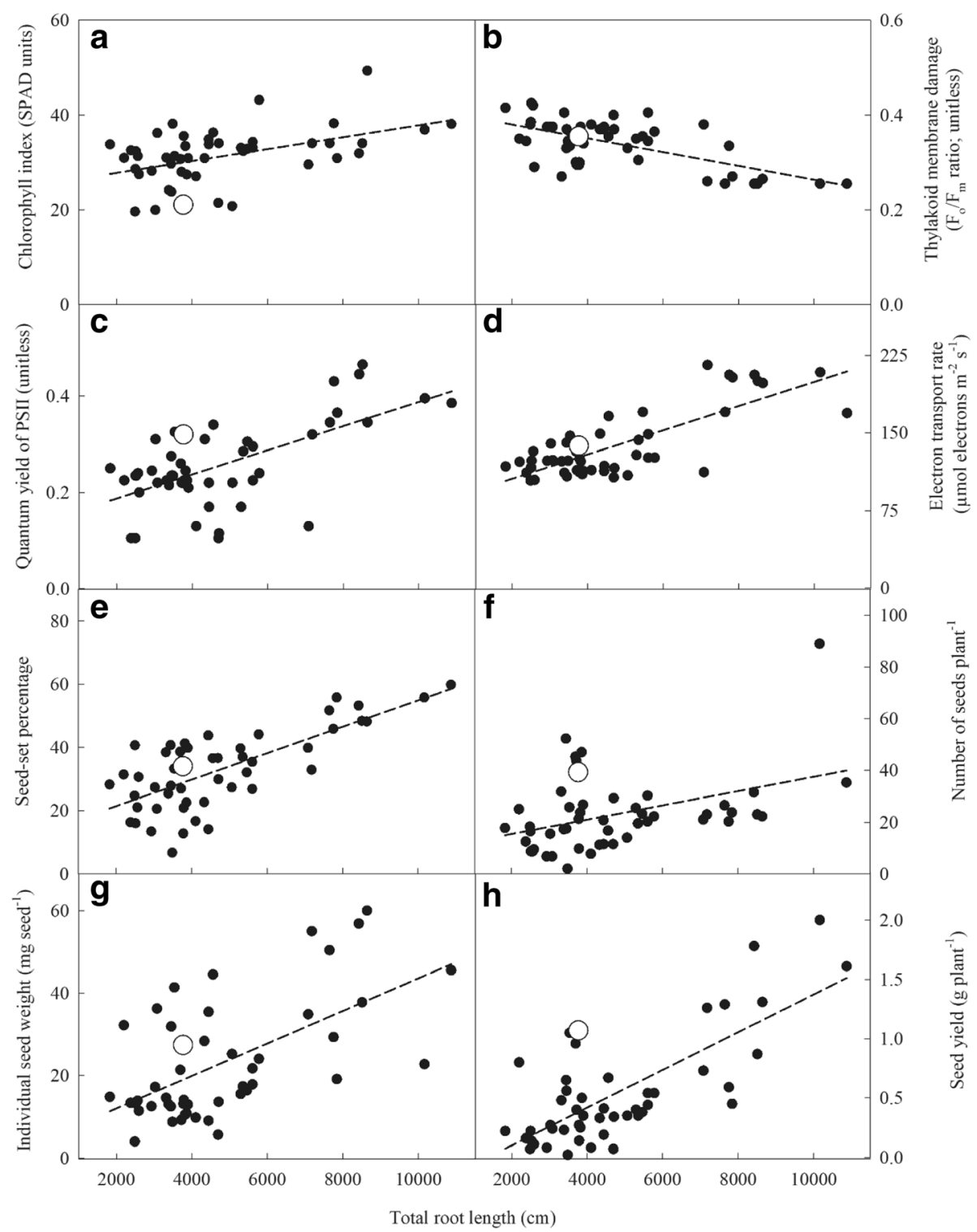

Fig. 2 Chlorophyll index (SPAD units), thylakoid membrane damage ( $F_{o} / F_{m}$ ratio; unitless), quantum yield of PSII (DPSIl; unitless), electron transport rate ( $\mu \mathrm{mol}$ electrons $\left.\mathrm{m}^{-2} \mathrm{~s}^{-1}\right)$, and components of seed yield per plant as a function of total root length $(\mathrm{cm})$ under drought stress in wheat-alien chromosome lines (water withheld for $16 \mathrm{~d}$ ). The CS check is shown as a white circle. The curve is fitted by a linear function. (a) chlorophyll index, $y=25.3+0.0013 x ; r^{2}=0.21(P<0.01)$; (b) thylakoid membrane damage, $y=0.409-0.000014 x ; r^{2}=0.39(P<0.01) ;(\mathbf{c})$ quantum yield of PSII, $y=0.137+0.000024 x ; r^{2}=0.39(P<0.01)$; (d) electron transport rate, $y=82.4+0.011 x ; r^{2}=0.60(P<0.01)$; (e) seed set percentage, $y=$ $13.083+0.0042 x ; r^{2}=0.52(P<0.001) ;(f)$ seed number per plant, $y=9.959+0.0028 x ; r^{2}=0.16(P<0.01) ;(\mathbf{g})$ individual seed weight, $y=4.142+$ $0.0039 x ; r^{2}=0.35(P<0.001)$ and $(\mathbf{h})$ seed yield per plant, $y=-0.217+0.0002 x ; r^{2}=0.54(P<0.001)$

Overall, the study suggests that photoinhibition of PSII under drought lead to reduced photochemical efficiency in the wheat-alien chromosome lines and the CS check.

Drought stress during flowering significantly reduced seed set percentage and individual seed weight in all wheat-alien chromosome lines (Table 7), which is attributed to the loss of gametic function and decreased seed filling rate and duration, respectively [47]. Significant genotypic differences were observed for seed set percentage and individual seed weight, which reflects the ability of the alien chromosome segment to endure drought. The wheat-alien chromosome lines TA5088 and TA5638 had higher seed set percentage and individual seed weight than the CS check. This is in accordance with the finding of Fang et al. [48] that drought-tolerant wheat genotypes had more seeds per plant and higher individual seed weights than drought-susceptible genotypes. 


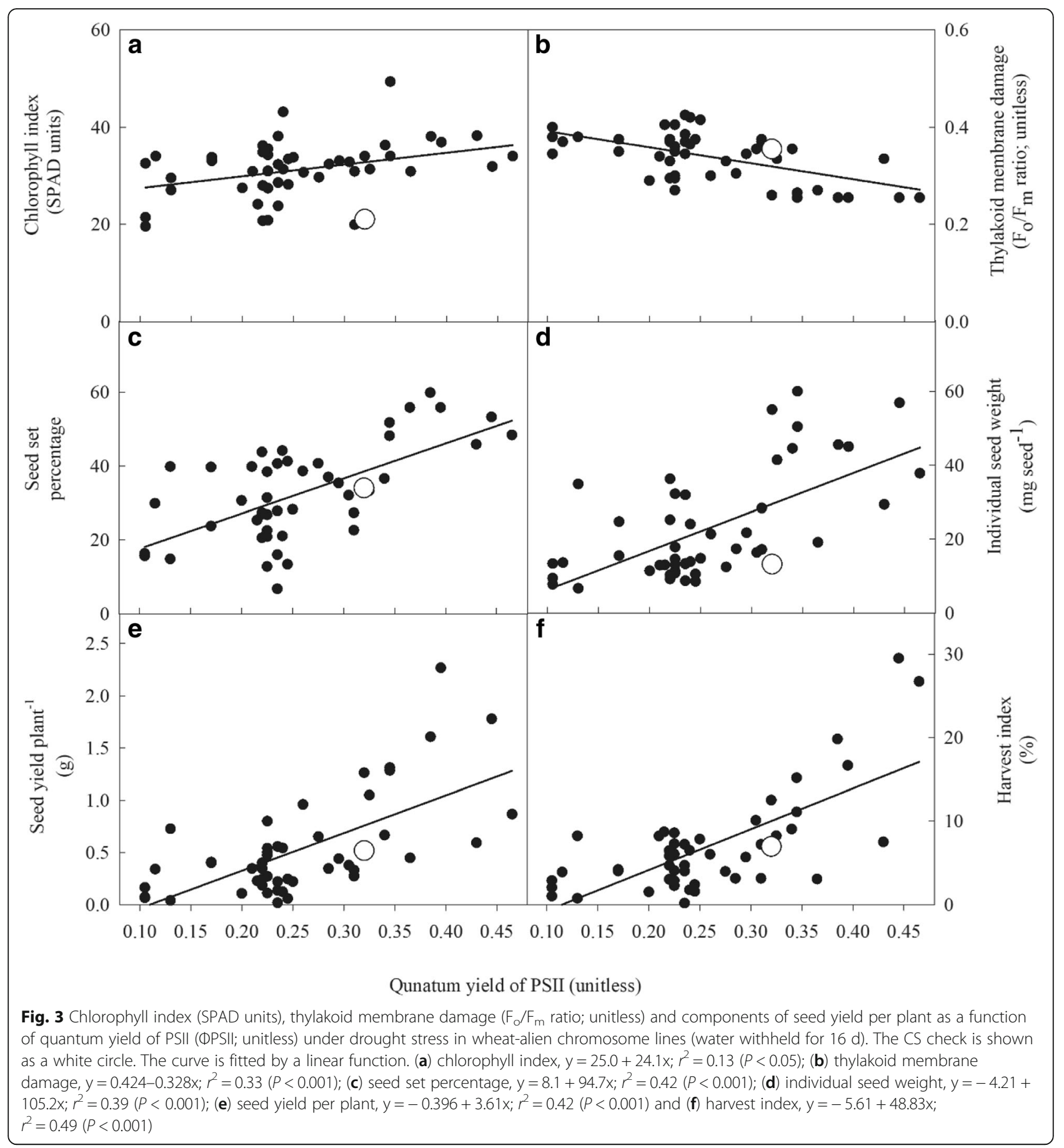

In wheat, the root biomass in subsoil correlates positively with individual seed weight and grain yield under drought stress [48]. Similarly, the ФPSII correlated positively with grain yield under drought stress [49]. The robust association of these variables with grain yield ratifies that yield is a function of water content and the photosynthetic process. The alien chromosome segments (5S of Ae. speltoides and $5 \mathrm{~V}$ of D. villosum) altered the root system and increased PSII photochemistry, which increased grain yield under drought stress. Two wheat-alien chromosome lines (TA5088 and TA5638) will be valuable germplasm for breeding for drought tolerance because of its deep root system and high reproductive success. Breeders can mobilize these translocated segments into adapted local germplasm and estimate the genetic value of these traits in their environment. It is possible that the same alien fragment introgressed into different wheat genotypes 
could exert different effects on resistance to drought. Therefore, the genetic background and interaction of these genetic factors need to be further investigated. Further, genetic and molecular studies need to be conducted to unravel the genetic factors controlling root- and shoot- related traits in the long arm of chromosome $5 \mathrm{~S}$ of Ae. speltoides and the short arm of chromosome $5 \mathrm{~V}$ of $D$. villosum, and their contribution to drought tolerance in wheat.

\section{Conclusions}

Alien chromosome segments altered root architecture and decreased photochemical efficiency, seed set percentage, individual seed weight, and seed yield per plant in 48 lines of wheat under drought. The wheat-alien chromosome lines TA5088 and TA5638, having chromosome or chromosome segment from Ae. speltoides (5S) and D. villosum $(5 \mathrm{~V})$, respectively, were identified as drought tolerant. These two lines had deep, thin and profuse root system under drought stress, which can help to alleviate drought stress by enhancing access to water. Total root length and the ФPSII were associated with higher seed set percentage and seed yield per plant under drought stress; hence, these traits can be used as proxy traits for improving drought tolerance in wheat. TA5088 and TA5638 lines will be valuable germplasm for understanding the molecular mechanism(s) and breeding of wheat for improved drought tolerance.

\section{Methods}

Seeds of 48 CS wheat-alien chromosome lines (chromosome addition/substitution /translocation) belonging to Ae. speltoides (4), Ae. searsii (3), Ae. longissima (5), Ae. peregrina (8), Ae. geniculata (14), Th. intermedium (3), L. racemosus (1) and D. villosum (10) and the background CS (check) were obtained from the Wheat Genetic Resources Center, Kansas State University, Manhattan, Kansas. The wheat-alien chromosome lines are derivatives of CS (T. aestivum)-Ae. speltoides, Ae. searsii, Ae. longissima, Ae. peregrina, Ae. geniculata, Th. Intermedium, $L$. racemosus and $D$. villosum. Details of the lines used in this study are in Additional file 1: Table S1.

\section{Experiment 1. Genetic variability in seedling root traits}

Twenty seeds of each of the 48 lines and the check were surface-sterilized using 10\% (v/v) sodium hypochlorite for $5 \mathrm{~min}$ and then washed with deionized water for three times. The seeds were germinated in Petri plates using filter paper (Whatman no 42) moistened with five $\mathrm{mL}$ of deionized water for two days. Square Petri plates $(12 \times 12 \times 1.7 \mathrm{~cm}, \mathrm{~L} \times \mathrm{W} \times \mathrm{H})$ was used in this study. A slit was made using a scissor on the sides of Petri plates top and bottom and covered with cellophane tape (Staples $^{\circ}$ Invisible Tape, $2 \times 3200 \mathrm{~cm}$, Staples, Manhattan,
KS). Sterilized agar (Sigma Type A; 2\% w/v) was poured into Petri plates. The Petri plates were sealed with cellophane tape. On the third day, uniformly sized seedlings (radicle emerged) were selected and placed one per Petri plates containing agar in the slit with the radicle facing downward. The Petri plates were incubated at $25 \pm 1{ }^{\circ} \mathrm{C}$ for $5 \mathrm{~d}$ [50]. After the stipulated time, the root angle of individual root axes of seminal roots, counting upwards from the primary seminal root (or radicle), was measured at $3 \mathrm{~cm}$ from the seed relative to a vertical line passing through the stem base [50]. Seedling root length was estimated using the cigar roll method [51].

\section{Experiment 2. Genetic variability in the shoot and root traits under drought}

The experiment was conducted in the greenhouse facilities at the Department of Agronomy, Kansas State University, Manhattan, KS to evaluate the variability of root system characteristics among 48 CS wheat-alien chromosome lines and a CS check. Before starting the experiment, the greenhouse was fumigated for one hour using an automated sulfur vaporizer (Rosemania, Franklin, TN) to avoid powdery mildew attack. The rooting medium was Turface MVP ${ }^{\circ}$ (PROFILE Products LLC, Buffalo Grove, IL), which had a bulk density of $577 \pm 32$ $\mathrm{kg} \mathrm{m}^{-3}$. Turface is a non-swelling illite and silica clay that allows easy separation of roots. Plants were grown in polyvinyl chloride (PVC) columns with an inside diameter of $7.5 \mathrm{~cm}$ and height of $150 \mathrm{~cm}$. The bottom of the PVC columns had plastic caps with a central hole of $0.5 \mathrm{~cm}$ diameter for drainage. Before sowing, each PVC column was filled with Turface and fertilized with $4 \mathrm{~g}$ of Osmocote (a slow-release fertilizer with 19:6:12 gravimetric percentages of $\mathrm{N}: \mathrm{P}_{2} \mathrm{O}_{5}: \mathrm{K}_{2} \mathrm{O}$; Scotts, Marysville, $\mathrm{OH}, \mathrm{USA}$ ) and $1 \mathrm{~g}$ of Marathon $1 \% \mathrm{G}$ (granular; a.i.: Imidacloprid:1-[(6-chloro-3-pyridinyl)methyl]-N-nitro-2-imidazolidinimine; OHP, Inc., Mainland, PA, USA) which were evenly mixed with the Turface in the top $2 \mathrm{~cm}$. Three seeds of a single genotype were sown at $4 \mathrm{~cm}$ depth in each PVC column. After emergence, the columns were thinned to one plant per column. Plants were maintained at $24 / 14{ }^{\circ} \mathrm{C}$ (daytime maximum/nighttime minimum temperature) from sowing to harvest (65 d after sowing) at a photoperiod of $16 \mathrm{~h}$ (natural light and supplemental fluorescent lights). The fungicide, Bumper 41.8 EC (emulsifiable concentrate; a.i.: Propiconazole:1-[[2-(2,4 dichlorophenyl)-4-propyl-1,3-dioxolan-2-yl]methyl]-1H-1,2,4-triazole; $1.2 \mathrm{~mL} \mathrm{~L}^{-1}$; Makhteshim Agan of North America, Inc., Raleigh, NC, USA) was applied on $20 \mathrm{~d}$ after sowing to prevent powdery mildew attack. The control plants were maintained at $100 \%$ field capacity from sowing to final harvest with drip irrigation. For the drought treatment, plants were stressed by withholding water from day 7 to final harvest ( 65 days). The duration of drought 
stress was 58 days at which the genotypes were at the booting stage (Feekes' stage 10). Our earlier experiments on wheat have indicated that plants grown in Turface have shown the drought stress effects (leaf rolling symptoms) after 45 days of stress imposition [52]. In another experiment withholding water for 41 days in common bean grown in Turface have decreased chlorophyll index by $5 \%$ and increased thylakoid membrane damage by $13 \%$ [53]. Therefore, in the present study water was withheld for 58 days (first drought symptoms of leaf rolling were observed about $45 \mathrm{~d}$ after withholding water) to cause a significant effect on biomass and root growth.

Plant height and number of tillers per plant were measured one day before harvest. Plant height was determined as the distance from the Turface level to the ligule of the youngest leaf and expressed in $\mathrm{cm}$. At harvest, the PVC columns were gently inverted at about $140^{\circ}$ to allow the contents (Turface and plants with entire root system) of the column to slip out. The shoots were cut at the base; and the aboveground biomass was oven dried at $65^{\circ} \mathrm{C}$ to constant weight, weighed, and expressed as g plant ${ }^{-1}$. The roots were carefully separated from the Turface without breaking the root system. The roots were laid on a flat surface and straightened to measure the maximum root length (rooting depth; from the base of the stem to the tip of the root system) and expressed in $\mathrm{cm}$. The root system was carefully washed in water to remove any adhering Turface, placed between the moist paper towels, sealed in Ziploc bags (S.C. Johnson \& Sons, Inc. Racine, WI, USA), transported to the laboratory, and stored at $4{ }^{\circ} \mathrm{C}$.

The root system of each plant was sliced into 30 -cm-long portions; each portion was submerged in water in a tray $(20 \times 15 \times 2 \mathrm{~cm} ; \mathrm{L} \times \mathrm{W} \times \mathrm{H})$, carefully spread to minimize root overlap, and scanned using an Epson photo scanner (Epson Perfection V700 with 600 dpi resolution, Epson, Long Beach, CA, USA). Images of scanned roots were analyzed using the WinRHIZO Pro image system (Regent Instruments, Inc., Quebec City, QC, Canada) to estimate total root length, root diameter and root volume as explained by McPhee [54] and Singh et al. [55]. Total root length, root diameter, and root volume were expressed in $\mathrm{cm}, \mathrm{mm}$ and $\mathrm{cm}^{3}$, respectively. The shoots were oven-dried at $60^{\circ} \mathrm{C}$ for $7 \mathrm{~d}$ to determine shoot dry weight. Maximum root length:shoot length ratio for each genotype was calculated as the ratio of maximum root length to plant height [56].

\section{Experiment 3. Genetic variability in physiological and yield traits under drought}

Experiment 3 was conducted to evaluate the variability in seed yield and its associated component traits among 48 CS wheat-alien chromosome lines and a CS check under drought conditions. Three seeds of a single genotype were sown in $1.6-\mathrm{L}$ plastic pots $[14 \mathrm{~cm}$ (height) $\times 50 \mathrm{~cm}$ (top perimeter) $\times 36 \mathrm{~cm}$ (bottom perimeter)] containing a 4:1 mixture of soil [well-drained Kennebec silt loam (a fine-silty, mixed, superactive, mesic Cumulic Hapludoll)] and sand, and $4 \mathrm{~g}$ of Osmocote Plus ( $\mathrm{N}: \mathrm{P}_{2} \mathrm{O}_{5}: \mathrm{K}_{2} \mathrm{O}=15: 9: 12$; Scotts, Marysville, $\mathrm{OH}$, USA). Two large indoor growth chambers (Conviron Model CMP 3244, Winnipeg, Manitoba, Canada) were used for this experiment, each being $136 \mathrm{~cm}$ wide, 246 $\mathrm{cm}$ long, and $180 \mathrm{~cm}$ high. Twenty-five lines were randomly placed within each growth chamber (24 CS wheat-alien chromosome lines and a CS check), with four pots per line. The growth chambers were maintained at $24 / 14{ }^{\circ} \mathrm{C}$ (daytime maximum/nighttime minimum temperature) from sowing to physiological maturity at a photoperiod of $16 \mathrm{~h}$ and $70 \%$ relative humidity $(\mathrm{RH})$, conditions that were optimal for the growth and development of the chromosome substitution lines [11]. The chambers were set at $70 \% \mathrm{RH}$ to avoid drought due to rapid evapotranspiration. Air temperature and $\mathrm{RH}$ were monitored at 20-min intervals in the growth chambers throughout the experiment. Photosynthetically active radiation (PAR) of $680 \mu \mathrm{mol} \mathrm{m}^{-2} \mathrm{~s}^{-1}$ was provided by cool white fluorescent lamps (Philips Lighting Co., Somerset, NJ, USA). The PAR was monitored once a month with a Field Scout Light Sensor (Spectrum Technologies, Inc., Plainfield, IL, USA). Fourteen days after seedling emergence, plants were thinned and staked, leaving two plants per pot. Granular Marathon 1\% (a.i.: Imidacloprid, 1-((6Chloro-3-pyridinyl) methyl)-N-nitro-2-imidazolidinimine) pesticide was applied to avoid infestation by sucking insect pests. Within a chamber, pots were randomly moved every $7 \mathrm{~d}$ to avoid any positional effects. The pots were kept in trays containing $2 \mathrm{~cm}$ of water to avoid drought; i.e., the plants were maintained at $100 \%$ pot capacity. At the booting stage, the main stem of each plant in the pots was tagged for recording yield and associated traits. Miracle-Gro, a water-soluble fertilizer $\left(\mathrm{N}: \mathrm{P}_{2} \mathrm{O}_{5}: \mathrm{K}_{2} \mathrm{O}=24: 8: 16\right.$; Scotts Miracle-Gro Products, Inc., Marysville, OH, USA) was added to the irrigation water (according to manufacturer's instructions) once in every $7 \mathrm{~d}$ until anthesis (Feekes' 10.5.1 stage). At anthesis, two pots of each line within each growth chamber were randomly assigned to the drought treatment. The drought stress was imposed by withholding water for $16 \mathrm{~d}$. The other two pots were continuously irrigated and served as the control. At the end of the stress, the plants were re-watered and kept well-watered until physiological maturity.

Various physiological traits viz., chlorophyll index, thylakoid membrane damage, ФPSII, and electron transport rate were measured on tagged plants on $12 \mathrm{~d}$ after the drought treatment. Chlorophyll index was measured with a self-calibrating chlorophyll meter (SPAD-502, 
Spectrum Technologies, Plainfield, IL, USA) on the fully expanded flag leaf of the tagged main stem and expressed in SPAD units. Each time, data were taken in triplicate from the middle portion of the leaf, and the readings were averaged. Chlorophyll $a$ fluorescence parameters were measured using a modulated fluorometer (OS-30p, Opti-Science Inc., Hudson, NH, USA). The minimal fluorescence $\left(\mathrm{F}_{\mathrm{o}}\right)$ and maximum fluorescence $\left(\mathrm{F}_{\mathrm{m}}\right)$ was measured in 30-min dark-adapted tagged flag leaves. Thylakoid membrane damage was determined as the ratio of $F_{o} / F_{m}$ (unitless). For other fluorescence measurements, the flag leaves were dark adapted for $2 \mathrm{~h}$; the leaves were then continuously irradiated with white actinic light to measure the initial fluorescence in leaves acclimated to irradiation $\left(\mathrm{F}_{\mathrm{o}}{ }^{\prime}\right)$, steady-state fluorescence yield $\left(\mathrm{F}_{\mathrm{s}}\right)$, and maximum fluorescence yield $\left(\mathrm{F}_{\mathrm{ms}}\right)$ of irradiated leaves. Using the above parameters, the ФPSII (ФPSII $=\left[\mathrm{F}_{\mathrm{ms}}-\mathrm{F}_{\mathrm{s}}\right] / \mathrm{F}_{\mathrm{ms}}$; unitless $)$ and apparent rate of photochemical transport of electrons through PSII $\left(\right.$ ETR $=$ ФPSII $\times$ PAR $\times 0.5 \times 0.84 ; \mu \mathrm{mol}$ electrons $\mathrm{m}^{-2} \mathrm{~s}^{-}$ $\left.{ }^{1}\right)$ were calculated using the instrument software [57, 58], where ETR is electron transport rate, PAR is incident photosynthetically active radiation on a leaf, 0.5 corresponds to the proportion of absorbed quanta used by PSII reaction centers, and 0.84 represents the proportion of incident irradiance absorbed by the leaf [59].

The spike from the main tiller was tagged at the heading stage. At physiological maturity, the tagged and remaining spikes were harvested separately and dried in an incubator at $40^{\circ} \mathrm{C}$ until constant weight. The vegetative biomass per plant was the weight of the oven dried $\left(65^{\circ} \mathrm{C}\right.$ for $\left.10 \mathrm{~d}\right)$ plant material without spikes and roots and expressed in gram. Individual spikelets were checked for grain by pressing the floret between the thumb and the index finger. Seed set percentage was estimated as the ratio of spikelets with grain to the total number of spikelets. The tagged spikes were hand threshed after drying, and the number of filled and unfilled grains were estimated for each spike. The remaining spikes were hand threshed, and the grains from these spikes counted and weighed to determine the number of grains per plant, grain weight per spike (g) and grain weight per plant (g). Individual grain weight was calculated by dividing grain weight per plant by number of grains per plant and expressed as $\mathrm{mg} \mathrm{seed^{-1 }}$. Harvest index (\%) was estimated as the ratio of grain yield to aboveground biomass.

\section{Data analyses}

Statistical analyses were performed with SAS 9.4 [60]. Experiment 1 had a completely randomized design with four replications, and Experiments 2 and 3 had a split-plot design in randomized complete block design with two replications. The main plots were water regimes and sub-plots were accessions. Experiments 1 and 3 were repeated. Data from experiment 1 and 3 and their corresponding repeats were statistically analysed independently and found that there were no significant differences. Therefore, the data from both the experiments were pooled together for combined statistical analyses and the mean responses are presented. The PROC GLM procedure of SAS was used for data analysis. Standard errors are shown as an estimate of variability, and the means of various variables are separated for significance by Fisher's least significant difference (LSD) at $5 \%$ significance level. The REG procedure in SAS was used to regress total root length and ФPSII against other traits. Principal component analysis (PCA) based on the correlation matrix, was performed using XLSTAT-Pro software (AddinSoft, Inc., NY, USA) to identify influential traits under drought stress [61]. PCA biplots were plotted for the drought stress conditions using XLSTAT-Pro software to show the relationships among studied genotypes based on recorded traits.

\section{Additional file}

Additional file 1: Table S1. List of wheat-alien chromosome lines and cultivars used in the present study [62-70]. (DOCX $17 \mathrm{~kb}$ )

\section{Abbreviations \\ CS: Chinese Spring; ETR: Electron transport rate; $F_{m}$ : Maximum fluorescence; $F_{\text {ms: }}$ : Maximum fluorescence yield; $F_{0}$ : Minimal fluorescence; $F_{s}$ : Steady-state fluorescence yield; LSD: Least significant difference; PAR: Photosynthetically active radiation; PC: Principal components; PCA: Principal component analysis; PSII: Photosystem II; PVC: Polyvinyl chloride; QTL: Quantitative trait locus; RH: Relative humidity; SPAD: Soil Plant Analysis Development; DPSII: Quantum yield of photosystem II}

\section{Acknowledgments}

The senior author (MD) thanks Tamil Nadu Agricultural University, India, for permitting him to perform post-doctoral research at Kansas State University, USA. Mention of trademark or proprietary product does not constitute a guarantee or warranty of the product by Kansas State University and does not imply its approval to the exclusion of other products, which may also be suitable. This publication is a Contribution Number 18-498-J from the Kansas Agricultural Experiment Station.

\section{Funding}

We thank the Triticeae Coordinated Agricultural Project (Triticeae-CAP), grant no. 2011-68002-30029 and 2017-67007-25939, from the USDA NIFA, United States Agency for International Development (USAID) Feed the Future Innovation Lab for Climate Resilient Wheat (Grant no. AID-0AA-A-13-00008); Feed the Future Innovation Lab for Collaborative Research on Sustainable Intensification (Grant no. AID-0AA-L-14-00006), and Kansas Wheat Commission. The funding from sponsors was used for experimental cost and publication fees. The experimental design, collection and analysis of data, interpretation of data and writing of this manuscript was carried out by the contributing authors.

Availability of data and materials

The data and materials are available from the corresponding authors upon request.

Authors' contributions

PWP and MD conceived and designed the experiments; MD and JK conducted the experiments. MD, SKS, ID, YC and KHMS analyzed the data. MD, PWP, and SKS wrote the manuscript: KHMS, YC, ID, BF and BSG edited the manuscript. BF and BSG provided genetic material. All authors read and approved the final manuscript. 


\section{Ethics approval and consent to participate}

Not applicable.

\section{Consent for publication}

Not applicable.

\section{Competing interests}

The authors declare that they have no competing interest.

\section{Publisher's Note}

Springer Nature remains neutral with regard to jurisdictional claims in published maps and institutional affiliations.

\section{Author details}

'Department of Agronomy, Kansas State University, Manhattan, Kansas 66506, USA. ${ }^{2}$ ICAR-National Bureau of Plant Genetic Resources, ICAR, New Delhi 110 012, India. ${ }^{3}$ Department of Agronomy, Horticulture and Plant Science, South Dakota State University, Brookings, SD 57007, USA. ${ }^{4}$ Wheat Genetic Resource Center, Department of Plant Pathology, Kansas State University, Manhattan, Kansas 66506, USA. Institute of Field and Vegetable Crops, Novi Sad, Serbia. ${ }^{6}$ The UWA Institute of Agriculture and School of Agriculture and Environment, The University of Western Australia, Perth, WA 6009, Australia. ${ }^{7}$ Department of Crop Physiology, Tamil Nadu Agricultural University, Coimbatore 641 003, India.

Received: 25 September 2018 Accepted: 15 May 2019 Published online: 07 June 2019

\section{References}

1. Ray DK, Mueller ND, West PC, Foley JA. Yield trends are insufficient to double global crop production by 2050. PLoS One. 2013;8(6):66428.

2. Dai A. Increasing drought under global warming in observations and models. Nat Clim Chang. 2013;3:52-8.

3. Ray DK, Gerber JS, MacDonald GK, West PC. Climate variation explains a third of global crop yield variability. Nature Comm. 2015;6:5989.

4. Placido DF, Campbell MT, Folsom JJ, Cui X, Kruger GR, Baezinger PS, et al. Introgression of novel traits from a wild wheat relative improves drought adaptation in wheat. Plant Physiol. 2013;161:1806-19.

5. Dvorak J, Terlizzi P, Zhang HB, Resta P. The evolution of polyploid wheats: identification of the a genome donor species. Genome. 1993;36:21-31.

6. Kihara H. Discovery of the DD-analyser, one of the ancestors of Triticum vulgare. Agric Hort. 1944;19:13-4.

7. Dvorak J, Zhang HB. Variation in repeated nucleotide sequences sheds light on the phylogeny of the wheat B and G genomes. PNAS. 1990;87:9640-4.

8. Valkoun JJ. Wheat pre-breeding using wild progenitors. Euphytica. 2001;119:17-23.

9. Liu W, Koo DH, Friebe B, Gill BS. A set of Triticum aestivum-Aegilops speltoides Robertsonian translocation lines. Theor Appl Genet. 2016:129: 2359-68.

10. Ehdaie B, Whitkus RW, Waines JG. Root biomass, water-use efficiency, and performance of wheat-rye translocations of chromosomes 1 and 2 in spring bread wheat 'Pavon'. Crop Sci. 2003:43:710-7.

11. Pradhan GP, Prasad PW. Evaluation of wheat chromosome translocation lines for high temperature stress tolerance at grain filling stage. PLoS One. 2015;10(2):e0116620.

12. Dvorak J, Edge M, Ross K. On the evolution of the adaptation of Lophopyrum elongatum to growth in saline environments. PNAS. 1988:85:3805-9.

13. McDonald MP, Galwey NW, Ellneskog-Staam P, Colmer TD. Evaluation of Lophopyrum elongatum as a source of genetic diversity to increase the waterlogging tolerance of hexaploid wheat (Triticum aestivum). New Phytol. 2001;151:369-80.

14. Monneveux P, Reynolds MP, Gonzalez-Aguilar J, Singh RP, Weber WE. Effects of the 7DL.7Ag translocation from Lophopyrum elongatum on wheat yield and related morphophysiological traits under different environments. Plant Breed. 2003;122:379-84.

15. Waines JG, Ehdaie B. Domestication and crop physiology: roots of greenrevolution wheat. Ann Bot. 2007;100:991-8.

16. Yediay F, Baloch F, Kilian B, Ozkan H. Testing of rye-specific markers located on 1RS chromosome and distribution of 1AL.RS and 1BL.RS translocations in Turkish wheat (Triticum aestivum L. durum Desf.) varieties and landraces. Genet Resour Crop Evol. 2010;57:119-29.
17. Yuan B, Cao X, Lv A. Gene introgression from common wheat into Aegilops L. Saudi J Biol Sci. 2017:24:813-6.

18. Friebe BR, Tuleen NA, Gill BS. Development and identification of a complete set of Triticum aestivum-Aegilops geniculata chromosome addition lines. Genome. 1999:42:374-80.

19. Sharma S, Bhat $P$, Ehdaie B, Close T, Lukaszewski A, Waines J. Integrated genetic map and genetic analysis of a region associated with root traits on the short arm of rye chromosome 1 in bread wheat. Theor Appl Genet. 2009;119:783-93.

20. Liang $H$, Yongliang $Y$, Hongqi $Y$, Lanjie $X$, Wei $D$, Hua $D$, et al. Inheritance and QTL mapping of related root traits in soybean at the seedling stage. Theor Appl Genet. 2014;127:2127-37.

21. Zhang H, Mittal N, Leamy LJ, Barazani O, Song BH. Back into the wild-apply untapped genetic diversity of wild relatives for crop improvement. Evol Appl. 2017;10:5-24.

22. Lukaszewski AJ. Reconstruction of complete chromosomes $1 \mathrm{~B}$ and $1 \mathrm{R}$ from the 1RS.1BL translocation of 'Kavkaz' origin. Genome. 1993;36:821-4.

23. Ehdaie B, Layne AP, Waines JG. Root system plasticity to drought influences grain yield in bread wheat. Euphytica. 2012;186:219-32.

24. Siddique KHM, Belford RK, Tennant D. Root: shoot ratios of old and modern, tall and semi-dwarf wheats in a Mediterranean environment. Plant Soil. 1990;121:89-98.

25. Wasson AP, Richards RA, Chatrath R, Misra SC, Prasad SV, Rebetzke GK, et al. Traits and selection strategies to improve root systems and water uptake in water-limited wheat crops. J Exp Bot. 2012;63:3485-98.

26. Comas LH, Becker SR, Curz VMV, Byrne PF, Dierig DA. Root traits contributing to plant productivity under drought. Frontiers in Plant Sci. 2013;4:442.

27. Uga Y, Sugimoto K, Ogawa S, Rane J, Ishitani M, Hara N, et al. Control of root system architecture by DEEPER ROOTING 1 increases rice yield under drought conditions. Nat Genet. 2013;45:1097-102.

28. Osipova S, Permyakov A, Permyakova M, Pshenichnikova T, Verkhoturov V, Rudikovsky $A$, et al. Regions of the bread wheat D genome associated with variation in key photosynthesis traits and shoot biomass under both wellwatered and water deficient conditions. J Appl Genet. 2016;57:151-63.

29. Bobo MS, Planchon C, Morris R. Chromosome 3D influences photosystem II quantum efficiency in winter wheat. Crop Sci. 1992;32:958-61.

30. Watanabe N, Ogawa A, Kitaya T, Furuta Y. Effect of substituted D genome chromosomes on photosynthesis rate of durum wheat (Triticum aestivum L. ). Euphytica. 1994;72:127-31.

31. Haour-Lurton B, Planchon C. Role of D-genome chromosome in photosynthesis expression in wheats. Theor Appl Genet. 1985;69:443-6.

32. Darko E, Janda T, Majlath I, Szopko D, Dulai S, Molnar I, et al. Salt stress response of wheat-barley addition lines carrying chromosomes from the winter barley "Manas". Euphytica. 2015;203:491-504.

33. P.V.V. Prasad, S.A. Staggenborg, Z. Ristic, Impacts of drought and/or heat stress on physiological, developmental, growth and yield processes of crop plants. In: "Response of Crops to Limited Water: Understanding and Modeling Water Stress Effects on Plant Growth Processes". (Eds L.H. Ahuja, S. A. Saseendran) (Advances in Agricultural Systems Modeling Series 1, ASACSSA: Madison, WI, USA) (2008) pp 301-355.

34. Saini HS, Aspinall D. Effect of water deficit on sporogenesis in wheat (Triticum aestivum L). Ann Bot. 1981;48:623-33.

35. Sharma S, Demason DA, Ehdaie B, Lukaszewski AJ, Waines JG. Dosage effect of the short arm of chromosome 1 of rye on root morphology and anatomy in bread wheat. J Exp Bot. 2010;61:2623-33.

36. Reynolds MP, Quilligan E, Aggarwal PK, Bansal KC, Cavalieri AJ, Chapman SC, et al. An integrated approach to maintaining cereal productivity under climate change. Global Food Security. 2016;8:9-18.

37. He XL, Wang JW, Li WX, Chen ZZ, Wu J, Zhao JX, et al. An intronless sucrose:fructan-6-fructosyltransferase (6-SFT) gene from Dasypyrum villosum enhances abiotic tolerance in tobacco. Biol Plant. 2017;61:235-45.

38. Yudina RS, Leonova IN, Salina EA, Khlestkina EK. Effect of alien genomic introgressions on the osmotic stress resistance of wheat. Russian J Genet Appl Res. 2015;5:168-73.

39. Rich SM, Watt M. Soil conditions and cereal root system architecture: review and considerations for linking Darwin and weaver. J Exp Bot. 2013;64:1193-208.

40. Manschadi AM, Christopher J, deVoil P, Hammer GL. The role of root architectural traits in adaptation of wheat to water-limited environments. Funct Plant Biol. 2006:33:823-37.

41. Chen YE, Su YQ, Zhang CM, Ma J, Mao HT, Yang ZH, Yuan M, Zhang ZW, Yuan S, Zhang HY. Comparison of photosynthetic characteristics and 
antioxidant systems in different wheat strains. J Plant Growth Reg. 2018;37 347-59.

42. Havaux M, Greppin H, Strasser RJ. Functioning of photosystems I and II in pea leaves exposed to heat stress in the presence or absence of light. Planta. 1991;186:88-98.

43. Tezara W, Marin O, Rengifo E, Martinez D, Herrera A. Photosynthesis and photoinhibition in two xerophytic shrubs during drought. Photosynthetica. 2005;43:37-45.

44. Pour-Aboughadareh A, Ahmadi J, Mehrabi AA, Etminan A, Moghaddam M, Siddique KHM. Physiological responses to drought stress in wild relatives of wheat: implications for wheat improvement. Acta Physiol Plant. 2017;39:106. https://doi.org/10.1007/s11738-017-2403-Z.

45. Bai ZY, Li CD, Zhao JF, Wu TY, Zheng JF, Bi CR, et al. Effect and preliminary analysis of chromosomal control on the chlorophyll fluorescence parameters of wheat substitution lines between synthetic hexaploid wheat and Chinese spring under drought stress. Sci Ag Sin. 2011;44:47-57 in Chinese.

46. Sairam RK, Shukla DS, Saxena DC. Stress induced injury and antioxidant enzymes in relation to drought tolerance in wheat genotypes. Biol Plant. 1997;40:357-64.

47. Alqudah AM, Samarah NH, Mullen RE. Drought stress effect on crop pollination, seed set, yield and quality. In: Lichtfouse E, editor. Alternative farming systems, biotechnology, drought stress and ecological fertilization. Dordrecht, the Netherlands: Springer; 2011. p. 193-213.

48. Fang Y, Du Y, Wang J, Wu A, Qiao S, Xu B. Moderate drought stress affected root growth and grain yield in old, modern and newly released cultivars of winter wheat. Front Plant Sci. 2017;8:672.

49. Paul K, Pauk J, Deak Z, Sass L, Vass I. Contrasting response of biomass and grain yield to severe drought in Cappelle Desprez and plainsman $V$ wheat cultivars. Peer J. 2016:4:e1708.

50. Manschadi AM, Hammer GL, Christopher JT, de Voil P. Genotypic variation in seedling root architectural traits and implications for drought adaptation in wheat (Triticum aestivum L.). Plant Soil. 2008;303:115-29.

51. Zhu J, Kaeppler SM, Lynch JP. Mapping of QTLs for lateral root branching and length in maize (Zea mays L.) under differential phosphorus supply. Theor App Genet. 2005;111:688-95.

52. Djanaguiraman M, Prasad PW, Kumari J, Rengel Z. Root length and root lipid composition contribute to drought tolerance of winter and spring wheat. Plant Soil. 2018. https://doi.org/10.1007/s11104-018-3794-3.

53. Sofi PA, Djanaguiraman M, Siddique KHM, Prasad PW. Reproductive fitness in common bean (Phaseolus vulgaris L.) under drought stress is associated with root length and volume. Ind J Plant Physiol. 2018;23:796-809.

54. McPhee K. Variation for seedling root architecture in the core collection of pea germplasm. Crop Sci. 2005;45:1758-63.

55. Singh V, van Oosterom EJ, Jordan DR, Hunt CH, Hammer GL. Genetic variability and control of nodal root angle in sorghum. Crop Sci. 2011;51: 2011-20.

56. Tomar RSS, Tiwari S, Vinod-Naik BK, Chand S, Deshmukh R, et al. Molecular and morpho-agronomical characterization of root architecture at seedling and reproductive stages for drought tolerance in wheat. PLOS One. 2016; 11(6):e0156528

57. Van Kooten O, Snel JFH. The use of chlorophyll fluorescence nomenclature in plant stress physiology. Photosynth Res. 1990;25:147-50.

58. Maxwell K, Johnson GN. Chlorophyll fluorescence- a practical guide. J Exp Bot. 2000;51:659-68.

59. Baker NR. Chlorophyll fluorescence: a probe of photosynthesis in vivo. Ann Rev Plant Biol. 2008:59:89-113.

60. SAS Institute. The SAS users guide. In: Version 9, vol. 3. USA: SAS Inst, Cary, NC; 2008.

61. Benson NU, Asuquo FE, Williams AB, Essien JP, Ekong Cl, et al. Source evaluation and trace metal contamination in benthic sediments from equatorial ecosystems using multivariate statistical techniques. PLoS One. 2016;11:e0156485.

62. Friebe B, Qi LL, Liu C, Gill BS. Genetic compensation abilities of Aegilops speltoides chromosomes for homoeologous B-genome chromosomes of polyploid wheat in disomic S(B) chromosome substitution lines. Cytogenet Genome Res. 2011;134:144-50.

63. Friebe B, Tuleen NA, Gill BS. Standard karyotype of Triticum searsii and its relationship with other S-genome species and common wheat. Theor Appl Genet. 1995:91:248-54.
64. Friebe B, Tuleen N, Jiang J, Gill BS. Standard karyotype of Triticum longissimum and its cytogenetic relationship with T. aestivum. Genome. 1993;36:731-42.

65. Friebe B, Tuleen NA, Badaeva ED, Gill BS. Cytogenetic identification of Aegilops peregrinum chromosomes added to common wheat. Genome. 1996b;39:272-6.

66. Koo H, Liu W, Friebe B, Gill BS. Homoeologous recombination in the presence of Ph1 gene in wheat. Chromosoma. 2017;126:531-40.

67. Liu C, Qi L, Liu W, Zhao W, Wilson J, et al. Development of a set of compensating Triticum aestivum-Dasypyrum villosum Robertsonian translocation lines. Genome. 2011:54:836-44.

68. Friebe B, Jiang J, Raupp WJ, McIntosh RA, Gill BS. Characterization of wheatalien translocations conferring resistance to diseases and pests: current status. Euphytica. 1996a;91:59-87.

69. Qi LL, Pumphrey MO, Friebe B, Zhang P, Oian C, Bowden RL, Rouse MN, Jin $Y$, Gill BS. A novel robertsonian translocation event leads to transfer of a stem rust resistance gene (Sr52) effective against race Ug99 from Dasypyrum villosum into bread wheat. Theor Appl Genet. 2011;123:159-67.

70. Qi LL, Pumphrey MO, Friebe B, Chen PD, Gill BS. Molecular cytogenetic characterization of alien introgressions with gene Fhb3 for resistance to Fusarium head blight disease of wheat. Theor Appl Genet. 2008;117:1155-16.
Ready to submit your research? Choose BMC and benefit from:

- fast, convenient online submission

- thorough peer review by experienced researchers in your field

- rapid publication on acceptance

- support for research data, including large and complex data types

- gold Open Access which fosters wider collaboration and increased citations

- maximum visibility for your research: over $100 \mathrm{M}$ website views per year

At $\mathrm{BMC}$, research is always in progress.

Learn more biomedcentral.com/submissions 